\title{
Informes
}

\section{Reinventando la cultura y el ocio en la era de la covid-19: buscando la oportunidad en medio de la crisis}

\author{
Reinventing culture and leisure in the age of covid-19: \\ seeking opportunity in the midst of crisis \\ C. Barbero Penas
}

\section{Resumen}

La llegada de la pandemia de covid-19 supuso el inicio de una nueva concepción del ocio y de la cultura dentro de los centros de la ONCE. Obligados a reinventarse por la necesidad, tanto los 22 centros como la Dirección de Promoción Cultural, Atención al Mayor, Juventud, Ocio y Deporte pusieron en marcha nuevas iniciativas y nuevas formas de llevar a cabo tanto actividades culturales como actividades de animación sociocultural y promoción deportiva. La realización de actividades telemáticas se erigió como alternativa más eficiente, permitiendo llegar a numerosos hogares e incluso aumentar el porcentaje de usuarios distintos en 2020 con respecto a años pasados. Estrechamente unidas a este auge de las Tecnologías de la Información y de la Comunicación (TIC), se realizaron actividades de todo tipo en los centros; paralelamente, desde la Dirección de Promoción Cultural, Atención al Mayor, Juventud, Ocio y Deporte, se potenciaron iniciativas como Cultura Global ONCE, y se implementaron otras nuevas como De libros y cine, el pódcast ONCINE, los talleres creativos online centralizados o la creación del canal de pódcasts Cultura ONCE. Todas estas acciones han contribuido a convertir esta crisis en una oportunidad, de tal forma que, incluso cuando se pueda retomar la actividad presencial con normalidad, es seguro que el formato de la cultura y el ocio será de carácter híbrido, con una combinación de actividad presencial y a distancia.

\section{Palabras clave}

Cultura. Ocio. Confinamiento. Innovación. TIC. 


\begin{abstract}
The onset of the covid-19 pandemic prompted new attitudes toward leisure and culture within the ONCE. Faced with the need for existential reinvention, the institution's 22 affiliate service centres, in conjunction with its Department of Furtherance of Culture, the Elderly, Youth, Leisure and Sport, undertook new initiatives and new ways to organise cultural activities and foster social-cultural coordination and participation in sport. Online activities proved to be the most effective alternative, as they attracted more user households in 2020 than in preceding years. The centres undertook all manner of information and communication technology (ICT)-related activities. In parallel, the Department of Furtherance of Culture, the Elderly, Youth, Leisure and Sport reinforced initiatives such as Cultura Global ONCE and endorsed new endeavours such as De libros y cine, a podcast entitled ONCINE, centralised creative online workshops and the creation of the Cultura ONCE podcast channel. All those measures have contributed to turning the crisis into an opportunity: even when in-person activities can be resumed, the cultural and leisure time offering will surely continue to be hybridised, combining in-person and remote activities.
\end{abstract}

\title{
Key words
}

Culture. Leisure. Lockdown. Innovation. ICTs.

La irrupción de la pandemia de covid-19, junto con la declaración del estado de alarma el 14 de marzo de 2020 y la consiguiente crisis sociosanitaria y económica marcaron el inicio de una nueva concepción del mundo del ocio, el tiempo libre, el deporte y la cultura. Inevitablemente, también dentro del ámbito de la ONCE se produjo un punto de inflexión y un hecho sin precedentes en toda la historia de la institución: la paralización casi total de toda la actividad de los centros de la ONCE. Ante esta situación, y en un contexto sociosanitario que difería de todo lo conocido anteriormente, no quedó más remedio que reinventarse y buscar, en ese momento más que nunca, nuevas formas de acercarse al colectivo de afiliados, muchos de los cuales se encontraban sufriendo situaciones de aislamiento y de soledad muy complicadas.

De este modo, y complementando la excelente labor realizada desde la Dirección Técnica de Bienestar Social, Prestaciones y Voluntariado, la Dirección de Promoción Cultural, Atención al Mayor, Juventud, Ocio y Deporte, junto con los Departamentos de Servicios Sociales para las Personas Afiliadas de todos los centros, realizaron un

Barbero, C. (2021). Reinventando la cultura y el ocio en la era de la covid-19: buscando la oportunidad en medio de la crisis. RED Visual: Revista Especializada en Discapacidad Visual, 77, 92-99. https://doi.org/10.53094/ DNSN5480. 
esfuerzo sin precedentes a fin de hacer llegar la cultura y el ocio a los hogares de las personas afiliadas. Así, desde el 14 de marzo hasta el 31 de diciembre se organizaron y llevaron a cabo un total de 1222 actividades (ver Tabla 1), un cifra muy meritoria, teniendo en cuenta que no fue hasta los meses de junio y julio cuando se comenzó a retomar cierta actividad presencial, bajo estrictas medidas de seguridad y siguiendo todos los protocolos sanitarios establecidos por la citada dirección ejecutiva que los 22 centros supieron adaptar a las realidades de cada comunidad autónoma, cada provincia e, incluso, de cada localidad.

Tabla 1. Distribución por tipología del número de actividades realizadas del 14 de marzo al 31 de diciembre de 2020

\begin{tabular}{|l|r|}
\hline \multicolumn{1}{|c|}{ Tipología de la actividad } & \multicolumn{1}{c|}{ N.o $^{-1}$} \\
\hline Culturales & 787 \\
\hline Promoción deportiva & 132 \\
\hline Recreativas & 112 \\
\hline Talleres creativos & 99 \\
\hline Talleres formativos & 92 \\
\hline TOTAL & $\mathbf{1 2 2 2}$ \\
\hline
\end{tabular}

¿Qué procesos se produjeron en la puesta en marcha de las propuestas culturales y de ocio y tiempo libre en esta nueva realidad? En primer lugar, el formato telemático - como era de esperar- cobró una importancia vital ya desde los inicios del confinamiento. La reacción de los departamentos de servicios sociales fue muy rápida en la reconversión de actividades a esta modalidad, pese a la situación de ERTE total o parcial que afectó a la mayor parte de los trabajadores de la ONCE -incluyendo los coordinadores de animación sociocultural y promoción deportiva- entre los meses de abril y junio. A pesar de las dificultades, se llevaron a cabo numerosas iniciativas: en la mayoría de los casos, el germen de la actividad online inició su andadura con el empleo del WhatsApp como medio de comunicación a través de mensajes, videollamadas o llamadas de voz, junto con las propias llamadas telefónicas. Al mismo tiempo en algunos casos o, poco después, en otros, irrumpieron populares plataformas como Zoom o Skype, que facilitaron una mejor comunicación y otorgaron a la persona afiliada una mayor sensación de cercanía, en gran parte también gracias a la admirable labor de los monitores. 
En estos primeros meses se realizaron numerosas tertulias telefónicas, reuniones de clubes de lectura o reuniones de cinefórum, empleando el uso del teléfono o a través de las plataformas ya mencionadas. Paralelamente, desde la Dirección se pone en marcha el pódcast ONCINE; esta iniciativa estaba prevista inicialmente para 2021, pero se consideró necesario adelantarla en el mes de abril de 2020, un momento en el que la población objetivo se encontraba ávida de contenidos sonoros que compartir y con los que suplir la falta de ocio presencial. Otro hito importante en el esfuerzo de hacer llegar los eventos más emblemáticos de la Dirección al colectivo de afiliados se produjo con la entrega de premios de la 33 edición de los Premios Tiflos, que, por primera vez en la historia, se realizó en formato virtual a través del canal de la ONCE en YouTube.

Es a partir de los meses de verano cuando se empiezan a retomar ya las propuestas de carácter presencial, combinándolas con el formato online para aquellas personas de riesgo que no viven cerca de los centros de la ONCE o que simplemente se sienten más cómodos y seguros realizando las actividades desde casa. Los coordinadores de animación sociocultural y promoción deportiva regresan al trabajo y llevan a cabo una labor extraordinaria, sucediéndose todo tipo de actividades a distancia y presenciales: eventos al aire libre, talleres literarios, gimnasia de mantenimiento, talleres de idiomas, encuentros virtuales, clases de cocina... y, por supuesto, numerosos talleres relacionados con las nuevas tecnologías en colaboración con los instructores de tiflotecnología y braille (ITB) de los centros de la ONCE, a fin de capacitar a todas las personas afiliadas interesadas en el uso de las TIC que han venido imponiéndose en el último año. Merece una mención especial el colectivo de mayores, que han mostrado una gran resiliencia y que se han adaptado rápidamente al empleo de las nuevas tecnologías de la información y de la comunicación por su cuenta, con ayuda de sus familiares o con el apoyo de los ITB.

Es en este momento de revitalización de las actividades socioculturales cuando cobra una mayor relevancia una iniciativa denominada Cultura Global ONCE, que, a lo largo 2020, se consolida como espacio para la comunicación con el colectivo de afiliados y no afiliados, con el pretexto de la cultura.

Cultura Global ONCE nace en mayo de 2019 con la finalidad de hacer llegar a todas las personas afiliadas diversas propuestas (presentaciones de libros, lecturas dramatizadas, charlas divulgativas, entrega de premios, etc.) que se organizan desde los diferentes centros de la ONCE, utilizando para ello las posibilidades de las tecnologías

Barbero, C. (2021). Reinventando la cultura y el ocio en la era de la covid-19: buscando la oportunidad en medio de la crisis. RED Visual: Revista Especializada en Discapacidad Visual, 77, 92-99. https:/doi.org/10.53094/ DNSN5480. 
de la comunicación y de la información. Si bien en el año 2019 se habían realizado ya cinco actividades, es en el último trimestre del año 2020 cuando se produce el despegue definitivo de propuesta abierta a todos los centros: se realiza un ciclo de charlas sobre ópera que obtiene enorme éxito entre el colectivo de afiliados, junto con una charla sobre ciberseguridad y dos tertulias con conocidos profesionales del mundo de la literatura y de la música, además de un taller de escritura creativa. Y es que el gran potencial de este programa ha permitido alcanzar nuevos horizontes territoriales, junto con la masa crítica necesaria para llevar a cabo iniciativas que, de otro modo, no podrían desarrollarse por diversas circunstancias.

Ante el incremento de propuestas a desarrollar dentro del programa Cultura Global $O N C E$, ha sido necesario realizar una reestructuración del proyecto y formalizar tanto los criterios para la realización de actividades como los requisitos técnicos necesarios que aseguren la máxima calidad en el desarrollo de la actividad y en su «puesta en escena». En este momento (marzo de 2021), se han realizado ya 2 eventos y se encuentran programados otros 9, por lo que podemos afirmar que se trata de un proyecto plenamente consolidado.

Dentro de este programa de Cultura Global ONCE nace en junio de 2020 De libros y cine (ver Figura 1). Se trata de una tertulia online de carácter intergeneracional, desarrollada en la plataforma Zoom, en la que cuatro personas afiliadas de distintos centros y edades debaten sobre una temática seleccionada a partir de una serie de libros y películas disponibles en la Biblioteca Digital de la ONCE (BDO) y en la videoteca de fondos audiodescritos (Audesc). Una vez grabada y editada, se pone a disposición del colectivo de afiliados en ClubONCE y a través de diversas plataformas de pódcast.

Figura 1. Imagen de la tertulia online De libros y cine

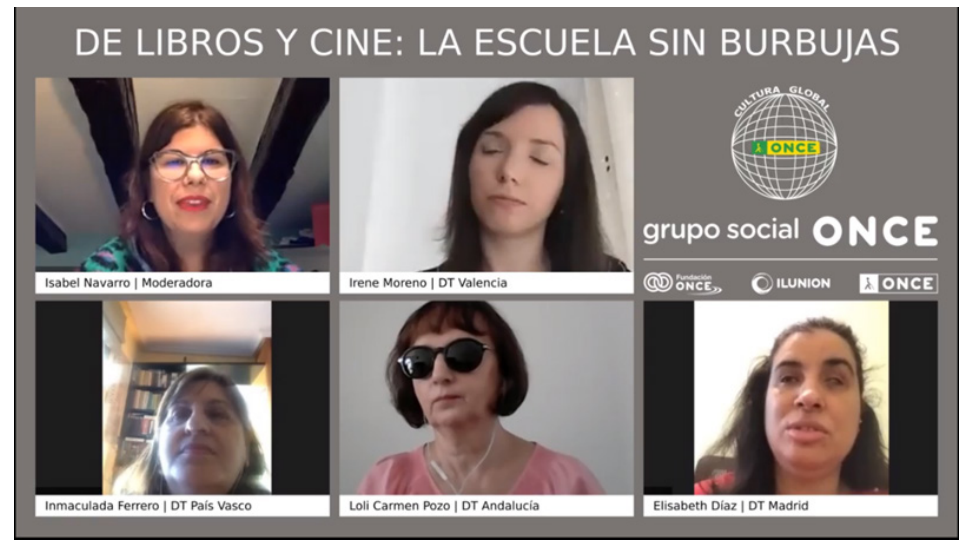

Barbero, C. (2021). Reinventando la cultura y el ocio en la era de la covid-19: buscando la oportunidad en medio de la crisis. RED Visual: Revista Especializada en Discapacidad Visual, 77, 92-99. https://doi.org/10.53094/ DNSN5480. 
Dentro del ámbito de acción de la Dirección de Promoción Cultural, Atención al Mayor, Juventud, Ocio y Deporte se encuentra también el Museo Tiflológico, una institución que, al igual que todos los museos del país, ha tenido que reimaginarse y reinventarse. Cerrado desde el día 13 de marzo hasta el 6 de julio, en los primeros meses de la pandemia se potenció la actividad en línea y se llevaron a cabo iniciativas como el Día de los Museos y la mesa redonda En lo tocante a tocar (ver Figura 2), al tiempo que se comenzaban a elaborar protocolos de seguridad y planes de contingencia listos para poner en marcha en el momento en el que el museo reabriera sus puertas.

Figura 2. Mesa redonda En lo tocante a tocar, organizada por el Museo Tiflológico y retransmitida en directo por YouTube

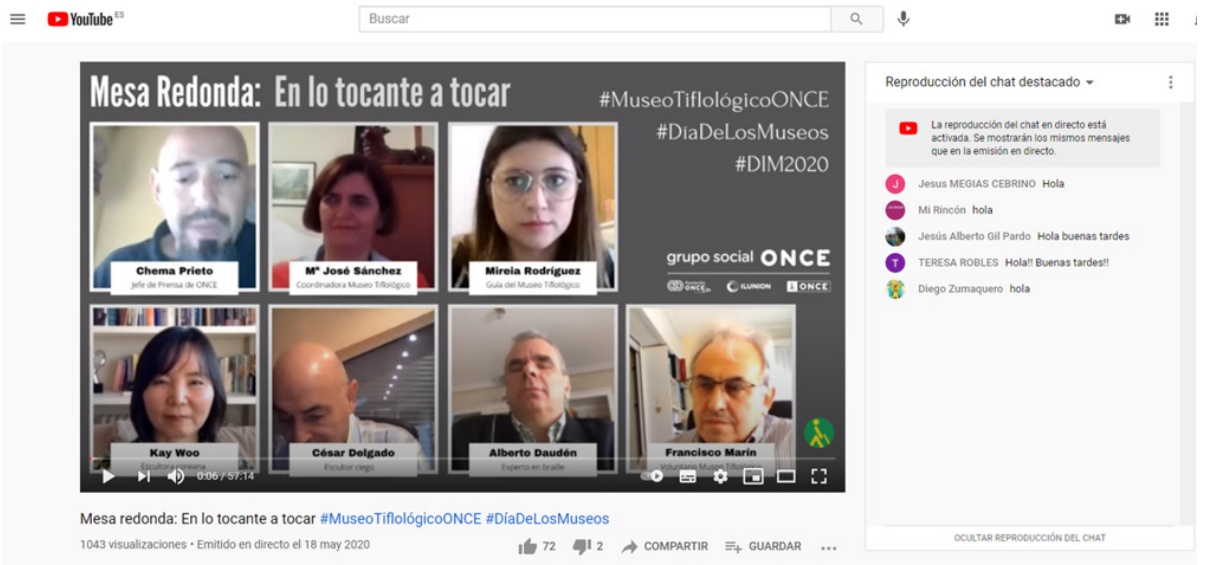

Respecto a los planes de prevención, el principal reto para el museo fue sortear las dificultades planteadas por la crisis sanitaria y que parecían hacer imposible la elaboración de un protocolo que permitiera a las personas ciegas seguir disfrutando del acceso táctil a las colecciones sin emplear guantes. A fin de preservar este derecho de acceso, se diseñó un plan especial en el que la higiene de manos y la supervisión directa de la exploración táctil fueran la clave, complementada por un período de cuarentena de 7 días para cada una de las piezas que recibiera algún tipo de contacto. El personal del museo juega un papel protagonista como garante de la seguridad, velando para hacer compatible la vocación de dar rienda suelta al tacto sin olvidar las medidas a las que obliga la pandemia. En el momento en el que se normalice la situación sanitaria, el objetivo será retomar el tacto como oportunidad de disfrute también para las personas sin discapacidad visual.

Otro cambio profundo en lo que respecta al museo ha sido lo referente a las visitas guiadas, tanto de colegios como de grupos: ahora es el museo el que va a 
los colegios mediante una charla-taller interactiva, y se realizan visitas guiadas y recorridos temáticos virtuales para los grupos que lo solicitan. Una de las ventajas que estas herramientas online han supuesto para el museo es que han facilitado la hipersegmentación del público, ofreciendo la posibilidad de realizar actividades más especializadas para grupos mucho más específicos. Este ha sido el caso de Manos a la obra: construyamos un templo, una actividad interactiva realizada a través de Teams y diseñada especialmente para los más pequeños, en la que se mezclaron diversas iniciativas del Departamento de Promoción Cultural y el Museo Tiflológico: proyección de un fragmento de una película audiodescrita, divulgación sobre una de las maquetas del museo (el Partenón) y una manualidad, para potenciar las habilidades artísticas de los más pequeños.

¿Qué ocurrirá en los próximos meses? Parece que al que podríamos denominar formato híbrido, puede aplicársele la expresión de moda de esta pandemia: «Ha llegado para quedarse». Es de esperar que, con la llegada de la vacuna y la disminución del riesgo de contagio de covid-19, sea posible en algún momento ir recuperando la presencialidad en el desarrollo de actividades de animación sociocultural y promoción deportiva, así como retomar las visitas presenciales grupales y escolares al Museo Tiflológico. Aun así, es improbable (y poco deseable) que el formato telemático desaparezca totalmente; si hay alguna buena noticia en cuanto a los usuarios de actividades del año 2020 es el aumento del porcentaje de usuarios distintos sobre el total de los usuarios de las actividades. Y es que el desarrollo de actividades online nos ha permitido llegar a numerosas personas afiliadas que no participaban en las actividades presenciales que ofertaban sus centros - por dificultades en los desplazamientos, lejanía de los centros en los que se desarrollaban, incompatibilidad de horarios, etc.-, pero que sí lo han hecho en ese formato virtual, convirtiéndose en heavy users de nuestro programa cultural y de ocio.

Por nuestra parte, seguimos trabajando para reinventarnos, para seguir aprovechando la oportunidad disfrazada de crisis que nos ha traído la pandemia de covid-19 y para seguir sorprendiendo al colectivo de afiliados con iniciativas frescas y diferentes dentro de nuestras posibilidades. Las últimas novedades en la Dirección son numerosas, destacando la retransmisión del fallo de la 34 edición de los Premios Tiflos en riguroso directo a través de YouTube, la fundación de la coral juvenil Staccato (que reúne a jóvenes de numerosos centros y que realizará ensayos a distancia), la creación del canal Cultura ONCE (ver Figura 3) -que se puede encontrar en diversas plataformas (Spotify, Google Podcasts, Apple Podcasts...) - y, finalmente, el estable-

Barbero, C. (2021). Reinventando la cultura y el ocio en la era de la covid-19: buscando la oportunidad en medio de la crisis. RED Visual: Revista Especializada en Discapacidad Visual, 77, 92-99. https:/doi.org/10.53094/ DNSN5480. 
cimiento del grupo de WhatsApp del Servicio de Audesc, que envía periódicamente a sus suscriptores información sobre los nuevos estrenos del catálogo y otras noticias de interés sobre audiodescripción.

Figura 3. Imagen del canal Cultura ONCE en Spotify

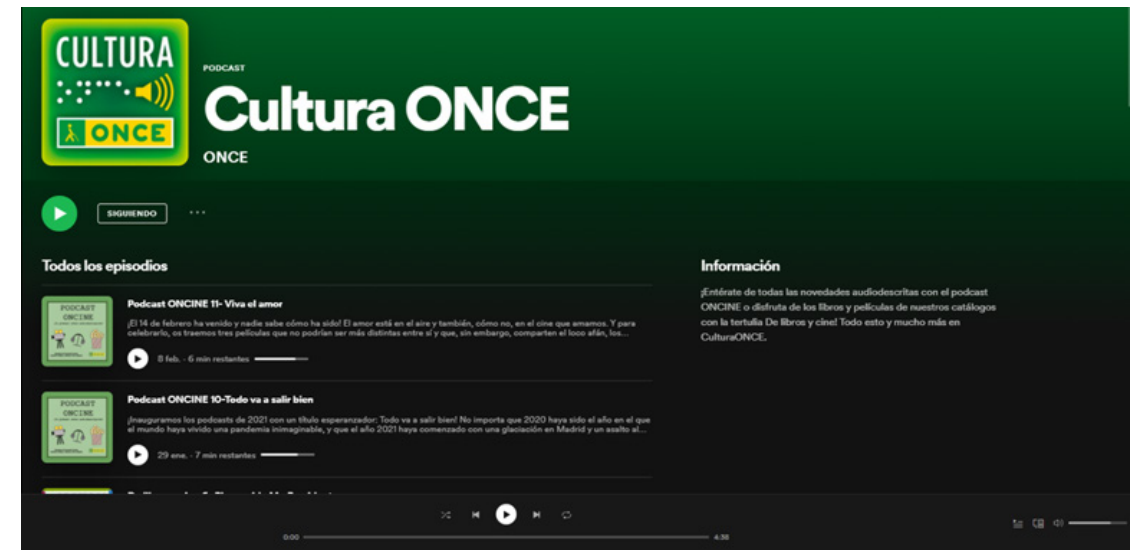

El futuro es incierto y, por eso mismo, supone un reto en el ámbito de la cultura, del ocio y del deporte, reto que aceptamos con ilusión, creatividad e innovación.

Clara Barbero Penas. Asesora técnica de la Dirección de Promoción Cultural, Atención al Mayor, Juventud, Ocio y Deporte. Dirección General de la ONCE. Calle del Prado, 24; 28014 Madrid (España).Correo electrónico: cbap@once.es. 